PONTIFÍCIA UNIVERSIDADE CATÓLICA DO RIO DE JANEIRO

\author{
Implicações do Alcoolismo \\ nas funções executivas
}

Regina Célia Rangel

Trabalho de Conclusão de Curso

\begin{abstract}
Centro de ClÊnCIAS SOCIAIS - CCS
Departamento de NeUROPSicologia
\end{abstract}

Pós Graduação em Avaliação Neuropsicológica 
Regina Célia Rangel

\section{Implicações do Alcoolismo nas Funções Executivas}

Trabalho de Conclusão de Curso

Trabalho de Conclusão de Curso, apresentado ao programa de pós-graduação em Avaliação Neuropsicológica da PUC-Rio como requisito parcial para a obtenção do título de especialista em Avaliação Neuropsicológica.

Orientadora: Prof ${ }^{a}$. Rosinda Oliveira

Rio de Janeiro agosto de 2018. 


\section{Resumo}

Rangel, Regina Célia. Implicações do Alcoolismo nas Funções executivas. Rio de Janeiro, 2018. Número de páginas 36 p. Trabalho de Conclusão de Curso - Avaliação Neuropsicológica. Pontifícia Universidade Católica do Rio de Janeiro.

O consumo de álcool é um problema de saúde pública com riscos à integridade do córtex pré-frontal, especialmente no concernente às funções executiva (FEs). As FEs podem ser definidas como um conjunto de processos cognitivos que inclui não só a tomada de decisões como outros processos mentais, que se mostram alterados no uso da substância álcool. O objetivo desse estudo foi fazer uma revisão a respeito das implicações do alcoolismo nas funções executivas. As principais alterações cognitivas encontradas em sujeitos adultos dependentes de álcool, relativas às FEs, ocorrem na tomada de decisão, controle inibitório e memória de trabalho, em paralelo a uma baixa ativação do córtex pré-frontal (CPF), encontrada mesmo após meses de abstinência.

Palavras-chave: Alcoolismo; Cognição; Funções Executivas; Córtex Préfrontal 


\section{SUMÁRIO}

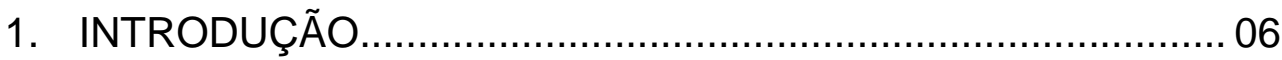

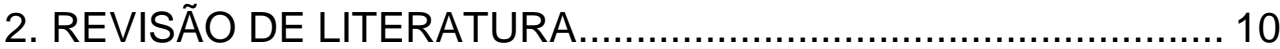

2.1 Evolução do Conceito de Alcoolismo........................................ 07

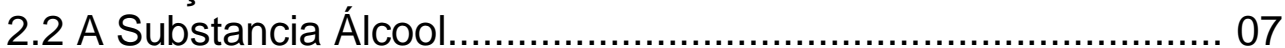

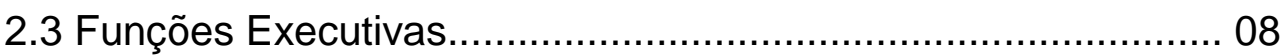

2.4 A Relação Alcoolismo e Funções Executivas............................ 09

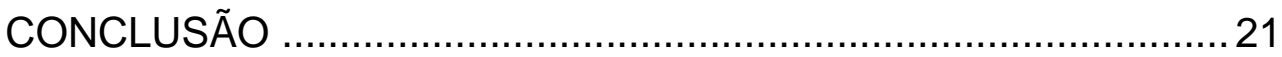

REFERÊNCIAS BIBLIOGRÁFICAS ............................................ 23 


\section{INTRODUÇÃO}

O consumo nocivo de álcool é um dos maiores problemas de saúde pública a nível mundial, sendo um fator de risco de morbidade e morte prematura.

Em nosso país, as bebidas alcoólicas são comercializadas livremente, sendo o álcool, considerado uma droga lícita (em muitos países é considerada ilícita). Isto contribui para que seja usado em larga escala, como facilitador de relações em eventos sociais, e aceito sem maiores problemas pela sociedade.

De acordo com a Organização Mundial da Saúde (WHO,2017) em 2016 o brasileiro bebeu mais álcool do que a média mundial. Dados revelam ainda que no Brasil o consumo per capita entre pessoas acima dos 15 anos foi de 8,9 litros, diante de 6,4 no restante do mundo. E ainda, segundo esses mesmos dados, milhões de mortes que ocorrem no mundo anualmente podem ser atribuídas ao uso dessa substância.

O alcoolismo é um transtorno de grande poder destrutivo e de alta prevalência (CARLINI, GALDURÓZ \& NOTO, 2001), relacionado a outras enfermidades e a comportamentos violentos, tais como agressões físicas, verbais e problemas com o sistema de justiça (ZHANG, WELTE \& WIECZOREK, 2002).

Muitos tipos de bebida contêm o álcool etílico, incluindo a cerveja, o vinho e diversos tipos de destilados, sendo que o teor alcoólico pode variar de 1,6\% na cerveja "light" a 40\% nos destilados como o gim e o uísque (WASHTON \& ZWEBEN, 2009).

Entre as substâncias psicoativas, o álcool é uma das mais consumidas, tanto pelo sexo feminino, como pelo masculino e cada vez mais na população jovem (adolescentes) (ANJOS et al., 2012).

A adolescência se caracteriza por um período de grandes mudanças tanto físicas, como fisiológicas, neurobiológicas, psicológicas e, ainda, de personalidade. Convém ressaltar, que essa fase é marcada caracteristicamente por busca de afirmação, acrescida do sentimento de onipotência, e de comportamentos impulsivos (RAPPAPORT, 1995). 
Assim, a procura de novas sensações, condução perigosa e, consequentemente, a experimentação de drogas e álcool, os potenciais de risco, se fazem presentes. Estes indivíduos jovens representam uma população de risco pelo alto potencial de se tornarem usuários dessas substâncias (BECHARA, 2001).

O consumo de álcool por indivíduos antes dos 15 anos, por exemplo, tem quatro vezes mais chances de desenvolver dependência de álcool na vida adulta e duas vezes e meia mais chances de se tronar abusadores de álcool do que aqueles que começam a beber após 21 anos (ESSAU \& HUTCHINSON, 2008).

Essa ocorrência se deve ao fato de que, nesse período o cérebro ainda encontra-se em processo de maturação, só atingindo a maturidade e desenvolvimento completo por volta dos 21 anos de idade (SCAIFE \& DUKA, 2009; JOHNSON et al., 2008). Por essa razão, os jovens são mais suscetíveis às alterações estruturais e funcionais no Sistema Nervoso Central (SNC), como a diminuição da plasticidade neuronal, do metabolismo, circulação sanguínea cerebral, atrofia, diminuição de volume da substancia cinzenta e branca que, conjuntamente conduzem aos déficits nas funções cognitivas (BASTOS,2014).

O álcool pode causar, em curto prazo, perturbações no funcionamento cerebral. Pequenas doses podem ocasionar dificuldades motoras, tempo maior de reação aos estímulos e decréscimos na memória; prejuízos reversíveis quando em sobriedade. Contudo, o uso prolongado ou em grandes quantidades tem potencial para gerar danos permanentes no tecido cerebral, resultando em prejuízos que persistem mesmo após um longo período de abstinência (NATIONAL INSTITUTE OF HEALTH, 2004).

Classificado como uma droga depressora do Sistema Nervoso Central, o álcool é capaz de afetar todas as funções do cérebro, o que inclui comportamento, cognição, discernimento, regulação da respiração, coordenação psicomotora e sexualidade. Além disso, o indivíduo pode afastarse de suas atividades usuais, familiares e de lazer, intensificando a freqüência das atividades que facilitam 0 uso (KAPLAN, SADOCK \& GREBB, 1997; WASHTON \& ZWEBEN, 2009). 
No Brasil, o alcoolismo é um importante problema de saúde pública, cujas consequências vão além dos prejuízos à saúde do consumidor, ocasionando amplas repercussões sociais (LARANJEIRA et al., 2007).

O consumo nocivo, no sujeito que usa a substância, pode levar a consequências físicas e doenças crônicas, como é o caso da cirrose e vários tipos de neoplasias. Além destas doenças, o consumo crônico e prolongado pode conduzir a doenças neurológicas, crônicas, como é o caso da Demência Persistente Induzida pelo Álcool e a Síndrome de Wernicke-Korsakoff, entre outras.

As técnicas de neuroimagem têm permitido investigações mais aprofundadas sobre as bases e natureza neurológica de determinadas patologias, propiciando o conhecimento da neurobiologia dos comportamentos, cognição e emoções, inclusive sobre o consumo e dependência do álcool.

Inúmeras evidências tem demonstrado o impacto negativo do álcool, tanto ao nível da estrutura, como na funcionalidade cerebral, relacionando intimamente as funções cognitivas mais afetadas, a essas alterações neuroanatômicas e neurofisiológicas (ROCHA et al.,2001).

A disfunção cognitiva ou déficits cognitivos presentes na população de consumo dessa substancia, pode acarretar uma série de prejuízos, principalmente déficits de aprendizagem e memória, capacidade visuoespacial, habilidades percepto-motoras, abstração e resolução de problemas (Parsons in Nassif \& Rosa, 2003), funções associadas às regiões frontal e fronto-têmporo-parietal (NASSIF \& ROSA, 2003).

Ainda, dentre as funções cognitivas, Uekermann et al. (2003) relata que na Sindrome de Dependência Alcoólica (SDA) as principais mudanças dizem respeito às funções executivas e à memória. Esses achados foram interpretados em termos de uma vulnerabilidade específica dos lóbulos frontais aos efeitos tóxicos do álcool (UEKERMANN, 2007).

Sujeitos dependentes dessa substância tendem a apresentar importantes alterações cognitivas, principalmente nas funções mnemônicas, atencionais e executivas, como por exemplo, na memória de trabalho; controle e seleção de resposta (intenção); resolução de problemas e tomada de decisões (ANDRADE, SANTOS, \& BUENO, 2004; CUNHA \& NOVAES, 2004). Tais prejuízos neurológicos poderiam contribuir para 
baixa adesão ao tratamento (VERDEJO-GARCIA, LOPEZTORRECILLAS, ARCOS \& PEREZ-GARCIA, 2005), o que torna importante conhecê-los.

Diante do exposto, o objetivo do trabalho em questão é realizar uma revisão teórica da relação entre Alcoolismo (Transtorno por uso de álcool) e Funções Executivas. Para tanto, serão analisados livros e artigos que abordam a referida temática. 


\section{REVISÃO DE LITERATURA}

\subsection{Evolução do conceito "Alcoolismo"}

A utilização do álcool remonta aos tempos pré-históricos e tem marcado todas as civilizações ao longo dos séculos, desempenhando inúmeras funções servindo de alimento, remédio ou, ainda, sendo empregado em ritos sociais, culturais e religiosos (EDWARD, MARSHALL, \& COOK, 1999).

De certa maneira, o "beber" sempre esteve associado ao status social, uma espécie de suporte às relações e às interações sociais. No entanto, junto a esse uso benéfico, também a "embriaguez" já se fazia notar (VIALA-ARTIGUES \& MECHETTI, 2003).

No século XVIII, as profundas mudanças estruturais da sociedade decorrentes da Revolução industrial, propiciam a produção e comercialização em larga escala de vários produtos, entre eles o álcool. A produção de bebidas em série, aumenta consideravelmente o número de consumidores e, por consequência, os problemas sociais causados pelo abuso no consumo do álcool, chamando a atenção para esse comportamento fora de padrão, e originando o conceito de alcoolismo.

Nesse movimento conceitual do alcoolismo, dois nomes se destacam. Thomas Trotter, por ter sido o primeiro a referir-se ao alcoolismo como doença; Benjamin Rush, psiquiatra americano pela frase "Beber inicia num ato de liberdade, caminha para o hábito e, finalmente, afunda na necessidade". Ambos caracterizaram a embriaguez como perda do autocontrole, a substancia passava a dominar o controle e a vontade do usuário (LARANJEIRA \& NICASTRI, 1996).

Em 1830 no EUA, o consumo médio era de 1,7 garrafas de bebidas destiladas por semana, três vezes mais do que a quantidade consumida em 2010. Nessa época consolidou-se a crença de que o consumo excessivo (a intemperança) constituía uma espécie de "pecado social".

Magnus Huss(1849), utilizou pela primeira vez o termo Alcoolismo, na tentativa de definir as complicações clínicas provenientes do uso abusivo e crônico de álcool. Parece surgir nessa época, a ideia de consumo 
nocivo do álcool relacionado à dependência, como algum tipo de doença ou distúrbio, aproximando-se do conceito atual (GOLD; ADAMEC, 2010; SOURNIA, 1986).

Na segunda metade do século $X X$, possibilitada pelo enfraquecimento dos discursos sobre a degenerescência, a compreensão do fenômeno "alcoolismo" relativo aos que usavam o álcool em larga escala, passa a ser visto sob uma ótica médica e consequentemente, passa a necessitar de critérios diagnósticos de maior confiabilidade e validade, sofrendo grande influência do notável trabalho de Jellinek, "The Disease Concept of Alcoholism", em que decreta o alcoolismo como doença (GIGLIOTTI \& BESSA, 2004).

A causa dessa doença seria fisiológica e identificável, própria a alguns indivíduos, e levaria à perda do controle volitivo e comportamental frente ao álcool inevitavelmente a partir do primeiro gole, fazendo surgir o conceito de dependência. No entanto, o diagnóstico do alcoolismo enquanto doença se restringia a situação na qual o usuário apresentasse tolerância, abstinência e perda do controle. Ressalta-se já aqui, o entendimento da tolerância, como sendo a exigência do organismo daquele que ingere o álcool, de doses cada vez maiores, para o mesmo efeito. Também como atualmente, a abstinência era entendida como síndrome, composta por um quadro de desconforto físico e/ou psíquico quando da diminuição ou suspensão de seu consumo, tal qual como atualmente. Nota-se assim já presente, uma diferenciação entre os transtornos por uso do álcool que envolviam um claro processo de dependência, daqueles sem o processo de dependência instalado (CONRAD; SCHNEIDER, 1992).

Em 1976, Grifith Edward e Milton Gross propuseram a Síndrome de Dependência do Álcool (SDA), se fazendo representar por um conjunto de sinais e sintomas comportamentais, fisiológicos e cognitivos. Nessa síndrome o uso do álcool alcança uma grande prioridade na vida de um indivíduo e as demais atividades passam a um segundo plano, caracterizando um relacionamento patológico entre a pessoa e a bebida (RAMOS \& BERTOLOTE, 1997).

Assim, ressalta-se como uma das principais características da SDA, proposta por Edwards, a distinção entre a categoria de abuso de álcool e 
outras drogas por padrões de uso patológico e prejuízos nas funções sociais e ocupacionais relacionados ao uso, e a categoria de dependência propriamente dita, que exigia além do uso patológico e prejuízos nas diversas funções, a presença de tolerância ou de abstinência (GIGLIOTTI \& BESSA, 2004).

Os conceitos da escola britânica de Grifith Edward serviram de base para os critérios diagnósticos da SDA, influenciou a elaboração dos principais códigos psiquiátricos como a Classificação Internacional das Doenças, 10ª versão (CID-10), publicada em 1992 pela Organização Mundial de Saúde (OMS), e o Manual diagnóstico e estatístico de transtornos mentais (DSM), da American Psychiatric Association (APA), desde o DSM III (1980) até o atual DSM V (2013) (GIGLIOTTI \& BESSA, 2004).

Atualmente, a dependência de álcool (alcoolismo) é entendida como uma doença crônica e multifatorial. Diversos fatores contribuem para o seu desenvolvimento, incluindo a quantidade e frequência de uso, a condição de saúde do indivíduo e fatores genéticos, psicossociais e ambientais. Apesar disso, o diagnóstico de dependência não é definido por esses fatores.

$\mathrm{Na} 10^{\mathrm{a}}$ edição da Classificação Internacional de Doenças (CID-10), da Organização Mundial da Saúde (OMS), a dependência é definida como um conjunto de fenômenos comportamentais, cognitivos e fisiológicos que se desenvolvem após o uso repetido de álcool. Este uso é tipicamente associado aos seguintes sintomas: forte desejo de beber, dificuldade de controlar o consumo (não conseguir parar de beber depois de ter começado), uso continuado apesar das consequências negativas, maior prioridade dada ao uso da substância em detrimento de outras atividades e obrigações, aumento da tolerância (necessidade de doses maiores de álcool para atingir o mesmo efeito obtido com doses anteriormente inferiores ou efeito cada vez menor com uma mesma dose da substância) e por vezes um estado de abstinência física (sintomas como sudorese, tremedeira e ansiedade quando a pessoa está sem o álcool).

Na 5aㅡ edição (2013), do Manual Diagnóstico Estatístico de Transtornos Mentais (Diagnostic and Statistical Manual of Mental Disorders, DSMV), da Associação Americana de Psiquiatria, os transtornos relacionados 
ao uso de álcool são definidos como a repetição de problemas decorrentes do uso do álcool que levam a prejuízos e/ou sofrimento clinicamente significativo. Sua gravidade varia de acordo com o número de sintomas apresentados.

A seguir são citados os Critérios para Dependência de Substâncias (adaptados da seção destinada aos transtornos para o uso de álcool do DSM-V Figlie et al., 2004). É necessário visualizar um padrão de uso disfuncional de uma substância, que leve a um comprometimento ou desconforto clinicamente significativo, no período de 12 meses consecutivos:

1. A substância é frequentemente consumida em grandes quantidades ou por um período maior do que o pretendido.

2. Há um desejo persistente ou esforços mal sucedidos para interromper ou controlar o uso.

3. Uma grande parte do tempo é gasta em atividades necessárias para obter a substância, usá-la ou recuperar-se de seus efeitos.

4. Presença de "fissura" ou um forte desejo ou urgência em relação ao uso da substância.

5. Uso recorrente da substância resultando na falha no cumprimento de obrigações importantes no trabalho, na escola ou no lar.

6. Uso contínuo da substância apesar de problemas interpessoais ou sociais, causados ou exacerbados por conta do uso da substância.

7. Atividades sociais, ocupacionais ou recreacionais importantes são abandonadas ou reduzidas em função do uso da substância.

8. Uso recorrente da substância em situações onde há prejuízo físico.

9. A substância é continuamente utilizada apesar do conhecimento da existência de problemas físicos ou psicológicos recorrentes ou persistentes, que são causados ou exacerbados pelo uso da substância.

10. Tolerância, definida por qualquer dos seguintes critérios:

a. Um desejo por quantidades marcadamente maiores para que intoxicação se manifeste ou para a obtenção dos efeitos desejados.

b. Uma diminuição clara dos efeitos observados ainda que se use a mesma quantidade da substância. 
11. Síndrome de abstinência, manifestada por qualquer dos seguintes aspectos:

a. Síndrome de abstinência característica da substância.

b. A mesma substância (ou outra bastante parecida) é utilizada para aliviar ou evitar os sintomas de abstinência.

Convém ressaltar, que enquanto o DSM-IV identificava duas condições diferentes, abuso de substância e dependência de substância, o DSM-5 une essas duas categorias em um continuum de gravidade do quadro no qual dependerá de quantos dos 11 critérios-sintomas (para determinadas substâncias) são atendidos, conforme abaixo:

1. Transtorno leve: Presença de 2 a 3 sintomas

2. Transtorno moderado: Presença de 4 a 5 sintomas

3. Transtorno grave: Presença de 6 ou mais sintomas

É possível perceber uma tendência maior de patologização do fenômeno no uso de substâncias, pois mesmo um uso moderado, com menor número de sintomas, passa a ser enquadrado como transtorno, ainda que leve. Outra mudança no DSM V, foi a inclusão do critério de fissura ("craving"), que é o forte desejo ou urgência em consumir a substância, sendo um dos principais riscos de recaída.

\subsection{A substância Álcool}

O álcool é uma substância psicoativa, ou seja, substancia que age principalmente no sistema nervoso central (SNC), lentificando seu funcionamento, sendo assim classificada como uma droga depressora. Vários sistemas neuroquímicos cerebrais sofrem alteração quando essa substancia é usada. Ao ser ingerido o álcool é facilmente absorvido pelo estômago para a corrente sanguínea e desta para o cérebro sem restrições, ativando o sistema de recompensa do cérebro (SRC) (LARANJEIRA et al., 2007; RIGONI et al., 2013).

Esse sistema é formado por circuitos neuronais responsáveis pelas ações reforçadas positiva e negativamente. Quando nos deparamos com um estímulo prazeroso, o cérebro lança um sinal: o aumento de dopami- 
na, neurotransmissor do sistema nervoso central (SNC), no núcleo accumbens, região central do sistema de recompensa e importante para os efeitos das drogas de abuso.

Segundo Feltenstein e See (2008), o processo de dependência em geral, ocorre como devido a entrada da droga no sistema de recompensa cerebral (SRC), via mesolímbica, no qual ocasiona uma desregulação desse sistema. Ressalta-se, no entanto, que apesar de cada droga de abuso ter o seu mecanismo de ação particular, todas elas atuam direta, ou indiretamente, ativando essa mesma região do cérebro, o SRC (LONGENECKER, 1998).

Independente da quantidade, o álcool age sobre os neurotransmissores (responsáveis pelas trocas de mensagens entre as células cerebrais), logo após a sua ingestão, especialmente sobre dois muito importantes para o comportamento humano: o ácido gama-aminobutírico (Gaba) e a serotonina. Esses transmissores se dividem em excitatórios e inibitórios. Os excitatórios estimulam a atividade elétrica do cérebro e, os inibitórios a reduzem. O álcool aumenta os efeitos do Gaba (neurotransmissor inibitório), o que causa os movimentos lentos e a fala enrolada que podem ser observados em pessoas alcoolizadas (PINEL, 2005; PLISZKA, 2004).

Concomitantemente, o álcool age inibindo o neurotransmissor excitatório glutamato, cancelando seus efeitos estimulantes, ocasionando certo retardamento fisiológico. Quando o sistema Gaba é acionado, e isso se dá, pela inibição da produção de glutamato, as pessoas se sentem mais relaxadas e com capacidade de interagir melhor com grupos. O que significa dizer que, quanto mais Gaba, menos autocontrole. Isso porque esse sistema é responsável pelo controle da ansiedade. Paralelamente, há também o aumento da liberação de serotonina, neurotransmissor regulador do prazer e do humor, capaz de proporcionar euforia, sendo que em alguns casos podem resultar em atos violentos (LARANJEIRA et al., 2007).

O uso abusivo de drogas,em geral, leva a sobrecarga do SRC, na medida em que, agradáveis sensações provenientes dessas substancias, motivam a ingestão cada vez maiores, potencializando a formação de as- 
sociações mal-adaptativas, gerando os sintomas clínicos, como abstinência, fissura, vulnerabilidade persistentes a recaídas (FUENTES et al., 2014)

Entre os abusadores crônicos de álcool, o nível de comprometimento encontra-se condicionado a quantidade e frequência do uso do álcool. Apesar de alguns manterem o nível intelectual praticamente intacto, outros apresentam comprometimentos que variam de alterações cognitivas leves, prejuízos moderados, podendo chegar a danos mais severos, como a demência persistente induzida pelo álcool e o transtorno amnéstico persistente induzido pelo álcool (Síndrome de Korsakoff). No entanto, convém ressaltar que mesmo nos denominados "bebedores sociais", cuja a ingestão se dá em torno de 21 doses por semana (cada dose equivale a $12 \mathrm{~g}$ de álcool), são encontrados indícios de alterações neurocognitivas em algumas funções mentais (CUNHA \& NOVAES, 2004).

Embora praticamente todas as estratégias terapêuticas para o alcoolismo apresentem resultados positivos, incluindo psicoterapias e inclusive grupos de mútua ajuda como o de Alcoólicos Anônimos (AA), muitos pacientes não apresentam melhora senão após várias tentativas fracassadas de tratamento ao longo dos anos.

Talvez uma das possíveis explicações para este aparente paradoxo encontre respaldo na observação dos danos causados pelo álcool, atuando principalmente no córtex pré-frontal. Desse dano decorre o prejuízo da tomada de decisões, fazendo com que o paciente escolha caminhos mais atraentes que the proporcionem um prazer imediato, ou mesmo busque alívio imediato de sintomas que estão causando sofrimento, como, por exemplo, seguir bebendo ao invés de manter-se abstinente (NASSIF \& ROSA, 2003; FUENTES et al., 2014).

Entre os processos mentais mais investigados e discutidos na literatura em neuropsicologia estão as funções executivas (FE), grupo de processos cognitivos que inclui não só a tomada de decisões como outros processos mentais, que se mostram alterados no uso da substancia álcool. 


\subsection{Funções Executivas}

Segundo a concepção neuropsicológica, as funções executivas (FEs) referem-se a um "conjunto de processos cognitivos que, de forma integrada, permitem ao indivíduo direcionar comportamentos a metas, avaliar eficiência e a adequação desses comportamentos, abandonar estratégias ineficazes em prol de outras mais eficientes e, desse modo, resolver problemas imediatos, de médio e de longo prazo" (MALLOY-DINIZ et al., 2008).

O dicionário da International Neuropsychological Society define funções executivas (FEs) como "as habilidades cognitivas necessárias para realizar comportamentos complexos dirigidos para determinado objetivo e a capacidade adaptativa às diversas demandas e mudanças ambientais" (LORING, 1999, P. 64).

Podem ser definidas, ainda, como um conjunto de habilidades que permitem ao indivíduo direcionar comportamentos para alcançar metas, resolver problemas imediatos, alternar comportamentos ou pensamentos de acordo com a exigência ambiental e inibir comportamentos inadequados em determinados contextos (GODOY, DIAS, TREVISAN, MENEZES, \& SEABRA, 2010; TONIETTO, WAGNER, TRENTINI, SPERB, \& PARENTE, 2011).

As funções executivas são processos cognitivos superiores que colaboram na manutenção de um arranjo mental apropriado para o alcance de um objetivo futuro, quando há necessidade do desempenho de processos de focalização atencional, inibição, gerenciamento de tarefas, planejamento e monitoramento na execução de um comportamento dirigido a objetivos (GRAFMAN, 1999; KRISTENSEN, 2006).

Podemos observar nas conceituações acima, que em geral, as funções executivas estão diretamente implicadas na capacidade de regulação do processamento da informação, envolvendo o controle voluntário e consciente por parte do indivíduo, podendo este escolher como agir sobre o meio que o circunda. Apresentam grande valor adaptativo, na medida em que possibilitam o "recalculo de rota", quando essa escolha no agir, se 
mostrar inadequada. O comprometimento dessas funções acarreta prejuízos para o indivíduo em tarefas desde as mais corriqueiras até as mais complexas, mesmo encontrando-se preservados outros domínios da cognição. Isto se dá em decorrência de tais funções serem responsáveis pela capacidade do indivíduo de autoregulação ou autogerenciamento.

Segundo Dias, Menezes e Seabra (2010), essas habilidades são especialmente relevantes diante de situações novas ou demandas ambientais que exijam ajustamento, adaptação ou flexibilidade.

Diversos processos cognitivos têm sido apontados como integrantes das funções executivas, tais como planejamento, controle inibitório, flexibilidade cognitiva, memória operacional, atenção, categorização, fluência, criatividade e tomada de decisão (PIEK, et al.,2004; ARNSTEN \& BAOMING, 2005; PAPAZIAN, ALFONSO \& LUZONDO, 2006; MALLOY-DINIZ et al.,2008).

Os lobos frontais constituem uma grande área cerebral responsável por múltiplas funções, o que determinou sua divisão em três regiões principais: córtex pré-central, córtex pré-motor, e córtex pré-frontal, sendo esse último mais relacionado às funções executivas (COSENZA, 2004; ROTTA, 2006; SANTOS, 2004).

As FEs são resultantes da atividade dos lobos frontais, especificamente das regiões pré-frontais, caracterizando-se por coordenar outros sistermas e estruturas neurais. Tal fato se deve a interconectividade única do córtex pré-frontal (CPF), que permite a coordenação e integração de outras estruturas cerebrais. Déficits nessas funções são verificados em indivíduos com comprometimentos que envolvem os circuitos pré-frontais, podendo assumir distintas formas de manifestação conforme o nível de acometimento dos circuitos envolvidos (MALLOY-DINIZ, 2010).

O circuito dorsolateral está relacionado a processos cognitivos de estabelecimento de metas, planejamento, solução de problemas, fluência, categorização, memória operacional, monitoração da aprendizagem e da atenção, flexibilidade cognitiva, capacidade de abstração, autorregulacão, julgamento, tomada de decisão, foco e sustentação da atenção (FUENTES et al., 2014). 
Segundo Fuster (2000), o córtex pré-frontal dorsolateral está envolvido em todas as formas de memória de trabalho em direção a um objetivo, seja no comportamento, raciocínio, ou na fala. Com papel central no controle, monitoramento e integração das atividades cognitivas, realiza ainda, a mediação da atenção no controle da distração, mantendo o foco, fornecendo flexibilidade para mudanças de contexto, estando também envolvido na geração de atividade verbal fluente e não-verbal (POWELL \& VOELLER, 2004).

O comprometimento desse circuito caracteriza-se pela incapacidade do indivíduo de manter a flexibilidade ou de mudar o contexto, mostrando comportamento rígido e perseverante, estratégias organizacionais pobres para tarefas de aprendizagem, bem como para copiar desenhos complexos e anormalidades de programação motora, mostrando deficiência em alternância de tarefas motoras recíprocas e dos testes motores sequenciais (CUMMINGS, 1993).

O circuito orbitofrontal é fortemente Interconectado com áreas de processamento cognitivo e emocional. Os processos cognitivos envolvidos são: o comportamento social, empatia, cumprimento de regras, controle inibitório, auto monitoração. Comprometimentos nessa região associam-se a comportamentos de risco, sensibilidade a normas sociais, infantilização, dependência de reforço evidente e baixa tolerância a frustração. Há também, prejuízo no julgamento social e no aprendizado baseado em emoções. $O$ paciente passa a apresentar dificuldades nos processos de tomada de decisão pela não antecipação de futuras consequências das atitudes. Damásio (1996) classifica esse último componente como "miopia para o futuro", e explica tais dificuldades a partir da hipótese dos marcadores somáticos (sensações corporais que sinalizam no processo de tomada de decisão). .

O circuito do cíngulo anterior é importante para a motivação, a monitoração de comportamentos, o controle executivo da atenção, a seleção e o controle de respostas. Comprometimentos nesse circuito podem levar a dificuldades na realização de atividades que requerem manutenção de respostas e controle da atenção. Como consequência do comprometimento dessa circuitaria, o paciente pode apresentar apatia, dificuldades 
em controlar a atenção, identificar e corrigir erros produzidos a partir de tendências automatizadas, desinibição de respostas instintivas e mutismo acinético.

Em resumo, as três áreas do córtex pré-frontal e suas conexões formam unidades funcionais, cada uma servindo a diferentes processos executivos (POWELL \& VOELLER, 2004).

As diversas manifestações conforme 0 acometimento nesses circuitos do CPF envolvidos, evidenciam que esse córtex não participa apenas nas operações executivas classicamente reconhecidas (sequenciamento, alteração, inibição, etc.), mas também desempenha papel central na coordenação da cognição e emoção (MITCHELL \& PHILLIPS, 2007). Essa ideia é corroborada por Ardila (2008), no caso de Phineas Gage que é geralmente citado como um típico exemplo de distúrbio de função executiva. Seus sintomas foram, na maioria, situados em um nível emocional/motivacional e não puramente cognitivo (ou metacognitivo). Essa dissociação observada incentivou alguns autores a classificarem as FEs em dois grupos, FEs "frias" e FEs "quentes" (BUELOW \& SUHR, 2009; CHAN, SHUM, TOULOPOULOU \& CHEN, 2008; SÉGUIN, ARSENEAULT, \& TREMBLAY, 2007).

As funções "frias" dizem respeito aos aspectos lógicos ou mecanicistas, componentes que não envolvem excitação emocional, como por exemplo, raciocínio lógico e abstrato, planejamento, resolução de problemas, memória de trabalho, inibição e flexibilidade cognitiva, entre outros. As "quentes", se referem aos aspectos emocionais. Estão envolvidas na regulação de comportamentos sociais, resoluções de conflitos que incluem fatores emocionais e interpessoais e comportamentos em que reforços e punições estão claramente em jogo (Chan et al., 2008), implicando na tomada de decisão. Depreende-se, portanto, a devastação no dia a dia de um indivíduo, proveniente de comprometimento dessas funções..

Ardila (2008) concorda com a participação do CPF nas FEs, entretanto, por reconhecê-las relacionadas, porém diferentes, propõe dois grupos de FEs: 1- referente as funções executivas classicamente reconhecidas como resolução de problemas, planejamento, formação de conceito, 
estratégia de desenvolvimento, controle da atenção e, memória de trabaIho e, 2- funções executivas de cunho emocional / motivacional.

Por ter um papel fundamental nos processos cognitivos mais complexos e na produção e controle do comportamento, o lobo pré-frontal é considerado o substrato neurológico mais envolvido nessas funções e diversos estudos de neuroimagem corroboram esta relação (GILMAN et al., 2010;GOUDRIAAN et al., 2006; MILLER \& WALLIS, 2009; ROCA et al., 2010). Esses estudos corroboram a deficiência do lobo frontal, como disfunção executiva com déficit de atenção e memória de trabalho, associada a incapacidade do indivíduo de abster-se à substancia álcool e, ás implicações clínicas negativas advindas desse uso, apontadas por Goldstein \& Volkow (2002).

\subsection{Relação Transtorno por uso de Álcool e Funções Executivas}

As pessoas acometidas pelo transtorno por uso de álcool apresentam alterações fisiológicas, comportamentais e cognitivas. No que tange aos aspectos cognitivos, as funções executivas tem papel central, considerando-se que, o indivíduo acometido por esse transtorno tende a apresentar importantes alterações, principalmente nas funções mnemônicas, atencionais e executivas, como por exemplo, na memória de trabalho; controle e seleção de resposta (intenção); resolução de problemas e tomada de decisões (ANDRADE, SANTOS, \& BUENO, 2004; CUNHA \& NOVAES, 2004).

Segundo Garcia et al.,(2014), as principais alterações cognitivas encontradas em sujeitos adultos dependentes de álcool, no concernente às FEs, ocorrem na tomada de decisão (CUNHA; NOVAES, 2004), controle inibitório (BARDENHAGEM; OSCAR-BERMAN; BOWDEN, 2007; CUNHA; NOVAES, 2004; HILDEBRANDT et al., 2004; KAREKEN et al., 2013; NOEL et al., 2007; ) e, memória de trabalho (CUNHA; NOVAES, 2004; HILDEBRANDT et al., 2004; THOMA et al., 2012). 
Ainda, alterações no CPF, especificamente no circuito orbitofrontal, são observadas, por uma baixa ativação, mesmo após meses de abstinência do álcool, provavelmente relacionado a problemas duradouros na atividade gabaérgica e serotoninérgica desta região, influenciando a tomada de decisão, controle inibitório e o comportamento de buscar novamente o álcool, mantendo assim, o processo de dependência (ABERNATH; CHANDLER; WOODWARD, 2010; CUNHA; NOVAES, 2004).

Em estudo quantitativo, com 90 pacientes com Síndrome de Dependência do Álcool (SDA), verificou-se prejuízo no desempenho Teste Wisconsin de Classificação de Cartas (WCST). Concluiu-se que os alcoolistas apresentam comprometimento das Funções Executivas, principalmente no que se refere à inibição de respostas (controle inibitório), fazendo um número maior de erros e perseverando mais nestes erros. Foi observada, também, dificuldade em aprender com a tarefa durante a execução da mesma (ARIAS et al., 2000; LANGLAIS \& CICCIA, 2000; PFEFFERBAUM et al., 2000; NASSIF \& ROSA, 2003; BECHARA et al., 2001).

Convém ressaltar que, alterações no controle inibitório também se fazem notar, quanto aos efeitos do uso eventual de álcool, mesmo na ausência de intoxicação pela substância (Ramaekers et al., 2011).

$\mathrm{Na}$ tomada de decisão, os Dependentes de substâncias psicoativas tendem a falhar em antecipar as consequências de seus comportamentos futuros. Segundo Bechara (2003), Tomassini et al., (2012), Kim et al., (2011) isso ocorre pela existência de dois tipos de funções executivas relacionados à dependência, que se distinguem pelo nível de incerteza em relação à resposta ou resultado: a decisão sob condições ambíguas e a decisão de risco.

As decisões sob condições ambíguas referem-se à decisão, entre diferentes opções, sem o conhecimento das respostas que sucedem, ou da possibilidade de recompensa ou punição (TOMASSINI et al., 2012; KIM et al., 2011). Podem envolver o circuito límbico, do processamento afetivo (TOMASSINI et al., 2012).

$\mathrm{Na}$ tomada de decisão considerada de risco, o indivíduo escolhe entre situações das quais já conhece o desfecho, ou seja, se são de recompensa ou punição (TOMASSINI et al., 2012; KIM et al., 2011). 
O processo de tomada de decisões tende a ser mostrar comprometido nos alcoolistas, em função das alterações no córtex pré-frontal, fazendo com que o paciente escolha caminhos mais atraentes que the proporcionem um prazer imediato, ou mesmo a busca de alívio imediato de sintomas que estão causando sofrimento, como, por exemplo, seguir bebendo ao invés de manter-se abstinente. $O$ paciente adota um comportamento sem levar em consideração as consequências futuras de suas atitudes (BECHARA et al.,2001).

Segundo Feldens et al.(2011), o alcoolismo é geralmente associado ao controle emocional prejudicado. Têm sido encontrados déficits nas funções executivas do lobo frontal, e estudos recentes com imagens funcionais têm sugerido que os alcoolistas apresentam maior ativação que os não-alcoolistas em circuitos envolvendo o lobo frontal, bem como em regiões posteriores do cérebro, durante a execução de tarefas. Isto foi observado, por exemplo, em tarefas em que os pacientes faziam julgamentos sobre locais e imagens que gostaram. Neste caso observou-se um aumento significativo da ativação nas regiões frontal, sistema límbico e temporal, nos pacientes em relação aos controles.

Goldstein e Volkow (2002), em estudo realizado objetivando avaliar o papel das estruturas do córtex pré-frontal na dependência química, concluíram que as áreas pré-frontais são ativadas em sujeitos durante a intoxicação, craving, bienging, sendo desativadas com a retirada da substância. Apontam ainda, que estas regiões estão envolvidas, também, nos níveis superiores de ordem cognitiva e motivacional, tais como a capacidade de acompanhar, atualizar e modular a evidência de um reforço da capacidade de controle prepotente de inibir as respostas.

Da mesma forma, Noel et al., (2007), ao comparar indivíduos dependentes do álcool recém desintoxicados e indivíduos não dependentes, utilizando o "Alcohol-Shifting Task", uma versão do GO/no GO, concluíram que os alcoolistas apresentam déficit na inibição da resposta, o que é reforçado quando a resposta a ser reprimida está relacionada ao álcool.

Noel e Bechara (2007), avaliaram 30 sujeitos dependentes do álcool e 30 indivíduos controle, utilizando o lowa Gambling Task (IGT) para investigar os vários processos de funções executivas. Os indivíduos com 
dependência do álcool apresentaram pontuação abaixo do normal para os últimos 20 ensaios do IGT. Além disso,também mostraram prejuízo em outras tarefas de funções executivas, especialmente aquelas que avaliam a capacidade de manipular informações armazenadas na memória de trabalho, detectar regras abstratas ou inibir respostas. Os resultados foram melhores quando os indivíduos tinham conhecimento sobre recompensas ou castigos da tarefa. Os autores enfatizam o importante papel que desempenha a inibição da resposta na tomada de decisão, especialmente em situações de risco.

Nos dependentes de álcool, além dos déficits na (inibição do comportamento) são ainda encontrados também déficits na memória de trabaIho,que se refere a um sistema envolvendo a memória de curto prazo,responsável pela manutenção e manipulação de informações na mente para a realização de tarefas cognitivas complexas (CUNHA; NOVAES, 2004).

Em estudo brasileiro com 152 alcoolistas, avaliou-se o desempenho no teste "Figuras Complexas de Rey". Os participantes que apresentaram dependência do álcool classificada como grave obtiveram diferenças estatisticamente significativas na pontuação da memória de trabalho, quando comparados àqueles com dependência moderada e aqueles que se mantiveram abstinentes apresentaram melhoras no escore da memória (OLIVEIRA et al., 2002).

Da mesma forma, o fraco desempenho dos pacientes com dependência de álcool, em termos de memória de trabalho, foi verificado por Crego e colaboradores (2010), em estudo no qual os sujeitos deveriam monitorizar uma série longa, de estímulos visuais, apresentados como dígitos, letras ou outro tipo de caracteres, que apareciam em intervalos regulares e os sujeitos deveriam responder quando observavam um alvo pré-designado.

Zinn et al.(2004), também observaram disfunção executiva em 27 alcoolistas, em termos de raciocínio abstrato, memória de trabalho e efetividade em testes que exigiam tempo de execução. Mostraram ainda, que a disfunção executiva pode persistir após a abstinência do álcool. Esse achado é corroborado com Kopera et al. (2012), com amostra de absti- 
nentes de álcool, em que verificaram uma diminuição da eficácia, numa tarefa de memória de trabalho, sugerindo que tal diminuição pode aumentar o risco de recaída.

De acordo com Cunha e Novaes (2004), pacientes dependentes de álcool, com alterações cognitivas e de neuroimagem, principalmente em regiões frontais do cérebro, tendem a apresentar pior prognóstico, associado a um maior número de recaídas durante o tratamento. Noel et al., (2001) avaliaram dependentes de álcool com testes neuropsicológicos ( controle inibitório, memória de trabalho, capacidade de abstração e memória verbal) e analisaram seu funcionamento cerebral através de tomografia computadorizada por emissão de fóton único (SPECT). Ao final de aproximadamente de 18 dias de abstinência (programa de desintoxicação), os pacientes apresentavam problemas no funcionamento neuropsicológico e cerebral, em relação aos controles. O controle inibitório e memória de trabalho se mostraram predominantemente alterados, tanto em termos comportamentais quanto de ativação de regiões frontais do cérebro. Os pacientes foram reavaliados dois meses depois e foi observado que, dos 20 pacientes dependentes, onze recaíram e nove se mantiveram na abstinência. Foi constatado que os pacientes recaídos, já na época da desintoxicação, mostraram pior desempenho na avaliação do controle inibitório, memória de trabalho e maiores alterações frontais em relação aos que se mantiveram abstinentes. 


\section{CONCLUSÃO}

Este estudo teve por objetivo pesquisar na literatura artigos que abordassem a temática sobre as implicações do alcoolismo nas funções executivas, em adultos.

Indivíduos alcoolistas, apesar de compreenderem os prejuízos associados ao uso de álcool e sofrerem consequências advindas deste, ainda assim mantêm um padrão crônico de consumo. Mesmo aqueles que buscam tratamento muitas vezes acabam por apresentar altas taxas de recaída. Estes aspectos no comportamento de pacientes alcoolistas são sugestivos de dificuldades que vão além de prejuízos de memória e atenção, indicando o envolvimento de déficits em funções executivas.

A breve revisão apresentada nesse trabalho sugere que pacientes usuários da substancia álcool apresentam alterações cognitivas associadas ao consumo, variando desde leves em abusadores, seguidas de prejuízos moderados em dependentes, podendo chegar a déficits neuropsicológicos mais graves.

Importante ressaltar que o uso esporádico do álcool era, no passado, considerado de baixo risco, sustentado pela crença de que por esse tipo de uso, não acarretaria consequências biológicas, psicológicas ou sócias. Hoje, indícios de alterações em algumas funções mentais, são reveladas, como por exemplo no controle inibitório e memória episódica verbal, mesmo nos bebedores ditos "sociais", ou seja, com uso eventual (WOICIK et al., 2009).

Nos dependentes de álcool, os comprometimentos se assemelham aos danos cerebrais difusos, persistindo por anos, mesmo após a ultima ingestão (CUNHA; NOVAES, 2004).

Sugere-se que os déficits cognitivos possam fazer parte de um padrão de funcionamento associado ao CPF, principal estrutura anatômica responsável pela função executiva, compreendida como um conjunto de capacidades cognitivas, necessárias, para o planejamento, início e regulação de um comportamento, para a obtenção de um objetivo (THUSH et al., 2008; GIANCOLA, 2007). 
Vários domínios neurocognitivos são integrados nas FE em termos funcionais tais como, inibição, flexibilidade cognitiva, tomada de decisão, capacidade atencional, planejamento de estratégias, resolução de problemas, raciocínio abstrato, formulação de hipóteses, sequenciação e aquisição de regras (HOUSTON et al., 2014; CREWS \& BOETTIGER, 2009; GIANCOLA, 2007). Importantes ainda, para a capacidade de organizar e utilizar a informação contida na memória de trabalho (GIANCOLA, 2007).

Nos pacientes acometidos pelo alcoolismo, as FE encontram-se em déficit, como demonstrado em vários estudos nessa revisão, sendo apontadas como as principais alterações, a tomada de decisão, o controle inibitório e a memória de trabalho.

A memória de trabalho tem papel fundamental na autorregulação e tomada de decisão. Indivíduos com diminuição da capacidade da memória de trabalho demonstram um menor controle dos impulsos (comportamento impulsivo), manifestam uma menor capacidade para reter e utilizar a informação e, por essa razão, escolhem comportamentos que geram a recompensa imediata. A relação entre a diminuição da autorregulação, comportamento impulsivo e menor capacidade da memória de trabalho, encontra-se na população dependente de álcool, como observada na presente revisão, apontada por diversos estudos.

Convém ressaltar ainda, que o aumento do consumo dessa substância entre jovens (adolescentes) coloca este grupo ainda em maior risco, considerando-se a intima relação do CPF e funções executivas.Quanto mais cedo o sujeito inicia o uso de álcool, mais prejuízos cognitivos poderá apresentar.

O CPF é das áreas cerebrais que atinge a maturidade e desenvolvimento completo mais tarde, cerca dos 21 anos de idade (SCAIFE \& DUKA, 2009; JOHNSON et al., 2008). Por essa razão, a população jovem é mais suscetível a desenvolver comportamentos e decisões consideradas de risco, quando comparada a adultos (Johnson et al., 2008). O desenvolvimento tardio dessa área pode ser especialmente sensível ao consumo do álcool. Estudos com adolescentes e jovens com padrão "Binge Drinking" verificaram, que este tipo de consumo, impacta negativamente o 
CPF, a partir da diminuição e degenerescência da substancia cinzenta e, consequentemente, afeta o controle cognitivo e emocional (SCAIFE \& DUKA, 2009; JOHNSON et al., 2008). Segundo Crego et al. (2010), adolescentes com este padrão de consumo manifestam maiores dificuldades em realizar tarefas que envolvem o CPF, como é o caso da memória de trabalho, planejamento, atenção e tomada de decisão. A disfunção do CPF acompanhado pelo déficit das funções executivas e controle emocional predispõem ao uso de substâncias, na população jovem (LYVERS et al., 2010).

Dessa forma, a experimentação de drogas, em geral, não deve ser relegada a segundo plano, como sendo "normal" durante a adolescência, exigindo acompanhamento em termos de avaliação das possíveis alterações em termos da atenuação ou da extinção com o tempo.

Apesar dos variados estudos sobre as implicações do alcoolismo nas FE representarem uma valiosa contribuição para a população em geral, sugere-se a realização de pesquisas que possam esclarecer ainda mais a questão central do alcoolismo no referente a tomada de decisão. Decidir de maneira disfuncional pode gerar diversos prejuízos ao longo da vida, já que esta habilidade é necessária cotidianamente. Conforme $\mathrm{Be}-$ chara et al. (2001), as alterações no processo de tomada de decisões podem fazer com que o paciente escolha seguir bebendo ao invés de manter-se abstinente, já que este não consegue refletir sobre as consequências futuras de seus atos.

Segundo Bertagnolli et al.,(2014), diversos danos são causados ao sistema nervoso por diferentes drogas. No entanto, não se sabe ao certo que parcela dos prejuízos na tomada de decisão pode ser anterior a dependência e que parcela pode ser resultado da neurotoxicidade da substância utilizada no sistema nervoso. Pode ser que dependentes de álcool sempre tenham tido prejuízos na tomada de decisão, prejuízos estes que os tornaram mais propensos a se tornarem alcoolistas.

Assim sendo, a necessidade de mais estudos, mostra-se relevante para a elucidação da etiologia desses prejuízos, que tanto prejudicam a adesão desses indivíduos a algum tipo de tratamento e, ainda favorecem a recaída e a manutenção da dependência a drogas em geral. 
Diante do exposto, a investigação neuropsicológica sobre as alterações associadas ao uso de substâncias, se faz imprescindível, para contribuir esclarecendo quais funções se encontram prejudicadas, bem como para estabelecer intervenções comportamentais para minimizar os prejuízos.

Além da descrição das alterações comportamentais, emocionais e cognitivas, a avaliação neuropsicológica busca realizar a análise de potenciais, prever o curso da recuperação e estimar o funcionamento prémórbido (anterior) dos usuários de substâncias psicoativas (GRUBER \& YURGELUN-TODD, 2001; ECKARDT \& MARTIN, 1986). É ainda do âmbito da neuropsicologia a realização de atividades que visem à recuperação ou amenização dos déficits neurocognitivos (reabilitação cognitiva), que se façam necessárias (ALLEN, GOLDSTEIN, \& SEATON, 1997). 


\section{REFERÊNCIAS BIBLIOGRÁFICAS}

ABERNATHY, K.E.; CHANDLER, L.J. \& WOODWARD, J.J. Alcohol and the prefrontal cortex. International review of neurobiology, 91, 289-320, 2010.

ALLEN, D. N.; GOLDSTEIN, G. \& SEATON, B. E. Cognitive rehabilitation of chronic alcohol abusers. Neuropsychology Review, 7, 21-39, 1997.

AMERICAN PSYCHIATRIC ASSOCIATION - APA. Diagnostic and statistical manual of mental disorders (DSM-5). 5.ed. Washington, DC, American Psychiatric Association, 2013.

AMERICAN PSYCHIATRIC ASSOCIATION (APA), Diagnostic and Statistical Manual of Mental Disorders, Fourth Edition. Washington, DC: American Psychiatric Association, 1994.

ANDRADE, V. M., SANTOS, F. H., \& BUENO, O. F. A. Neuropsicologia hoje. São Paulo: Artes Médicas, 2004.

ANJOS, KARLA \& SANTOS, VANESSA \& ALMEIDA, OBERTAL. Perfil do consumo de bebidas alcoólicas por adolescentes escolares. Rev.Saúde.Com, 8(2): 20-31, 2012.

ARDILA, A. On the evolutionary origins of executive functions. Brain and Cognition, 68, 92-99, 2008.

ARIAS, J.L.; SANTIN, L.J. \& RUBIO, S. Effects of chronic alcohol consumption on spatial reference and working memory tasks. Alcohol, 20, 149-159, 2000.

ARNSTEN, A. F.T. \& BAO-MING, L. Neurobiology of Executive Functions: Catecholamine Influences on Prefrontal Cortical Functions. Biological Psychiatry, Volume 57, $1377-1384,2005$.

BARDENHAGEN, F. J.; OSCAR-BERMAN, M, ; BOWDEN, STEPHEN C. Rule knowledge aids performance on spatial and object alternation tasks by alcoholic patients with and without Korsakoff 's amnesia. Neuropsychiatric disease and treatment, v.3, n.6, p.907, 2007.

BASTOS, A. Estimulação Cognitiva em Pacientes com Alcoolismo: Vantagens da Utilização de Dispositivos Móveis. 2014.156 f. Dissertação (Mestrado em Neuropsicologia) - Universidade Lusófona de Humanidades e Tecnologias, Lisboa. 2014.

BECHARA, A., DOLAN, S., DENBURG, N., HINDES, A., ANDERSON, S.W.\& NATHAN, P.E. Decision-making deficits, linked to a dysfunctional ventromedial prefrontal cortex, revealed in alcohol and stimulant abusers. Neuropsychologia, 39, 376-89, 2001. 
BECHARA, A. Risky business: Emotion, decision-making, and addiction. Journal of Gambling Studies, 19, 23-51, 2003.

BERTAGNOLLI, ANA CRISTINA; KRISTENSEN, CHRISTIAN HAAG; BAKOS, DANIELA SCHNEIDER. Dependência de álcool e recaída: considerações sobre a tomada de decisão. Aletheia, Canoas, n. 43-44, p. 188-202, ago. 2014.

Disponível em http://pepsic.bvsalud.org/scielo. php?script=sci_arttext\&pid=S1413-

$03942014000100014 \& \operatorname{lng}=$ t\&\&nrm=iso $>$. acesso em 28 set. 2018.

BUELOW, M. \& SUHR, J. Construct Validity of the lowa Gambling Task. Neuropsychology review, 19, 102-14, 2009.

CARLINI, E.A.; GALDURÓZ, J.C. \& NOTO, L. I Levantamento Domiciliar Sobre o Uso de Drogas Psicotrópicas no Brasil - 2001. São Paulo: CEBRID.

CHAN, R. C. K., SHUN, D., TOULOPOULOU, T., CHEN, E. Y. H. Assessment of executive functions: Review of instruments and identification of critical issues. Archives of Clinical Neuropsychology, 23, 201-216, 2008.

CONRAD, P.; SCHNEIDER, J. W. Deviance and medicalization. From badness to sickness. Philadelphia: Temple University, 1992.

COSENZA, R. M. (2004). Bases estruturais do sistema nervoso. In V. M. ANDRADE; F. H. SANTOS \& O. BUENO (Eds). Neuropsicologia Hoje. São Paulo, Artes Médicas, p. 37-59, 2004.

CREGO, A.; RODRIGUEZ-HOLGUÍN, S.; PARADA, M.; MOTA, N.; CORRAL, M. \& CADAVEIRA, F. Reduced anterior prefrontal cortex activation in young binge drinkers during a visual working memory task. Drug and Alcohol Dependence, 109: 45-56, 2010.

CREWS, F.T. \& BOETTIGER, C.A. Impulsivity, frontal lobes and risk for addiction. Pharmacology, Biochemistry and Behavior, 93, p. 237-247, 2009.

CUMMINGS, J.L. Frontal-subcortical circuits and human behavior. Arch Neurol, 50, 873-880, 1993.

CUNHA, P. J. \& NOVAES, M. Avaliação neurocognitiva no abuso e dependência do álcool: Implicações para o tratamento. Revista Brasileira de Psiquiatria, 26 (Supl I), 23-27, 2004.

DAMÁSIO, A. R. O erro de Descartes: emoção, razão e cérebro humano. São Paulo, Companhia das Letras, 1996. 
DIAS, N. M.; MENEZES, A. \& SEABRA, A. G. Alterações das funções executivas em crianças e adolescentes. Estudos Interdisciplinares em Psicologia, 1(1), 80-95, 2010.

ECKARDT, M.J.; MARTIN, P.R. Clinical Assessment of Cognition in Alcoholism. Alcoh Clin Exp Researc, v. 10, n. 2, p. 123-7, 1986.

EDWARDS, G., MARSHALL, E.J. \& COOK, C.C.H. O tratamento do alcoolismo: um guia para profissionais de saúde ( $3^{\underline{a}}$ ed.). Porto Alegre: Artes Médicas, 1999.

ESSAU, C.A. \& HUTCHINSON, D. (2008). Alcohol Use, Abuse and Dependence. In: C.A. ESSAU (Ed.). Adolescent Addiction: Epidemiology, Assessment and Treatment. (1 ${ }^{\mathrm{a}} \mathrm{Ed}$., pp. 61-115) London: Elsevier Inc., 2008. P. 61-115.

FELDENS, A. C. M., SILVA, J. G., \& OLIVEIRA, M. S. Avaliação das funções executivas em alcoolistas. Cadernos de Saúde Coletiva, 19, 164$171,2011$.

FELTENSTEIN, M. W.; SEE, R.E. (2008). The neurocircuiyty of addiction: an overview. Br.J. Pharmacol. 154, 261-274, 2008.

FIGLIE, N. B.; LARANJEIRA, R. \& BORDIN, S. Aconselhamento em dependência química. São Paulo: Roca, 2004.

FUENTES, D. et al. Neuropsicologia: teoria e prática. 2. ed. Porto Alegre, Artmed. 2014.

FUSTER, J. M. Cortex and mind: unifying cognition. New York, Oxford University Press, 2003.

GIANCOLA, P.R. The underlying role of aggressivity in the relation between executive functioning and alcohol consumption. Addictive Behaviors, v. 32, n. 4, p. 765-783, 2007.

GIGLIOTTI, A. \& BESSA, M. A. Síndrome de dependência do álcool: Critérios diagnósticos. Revista Brasileira de Psiquiatria, 26, 11-13, 2004.

GILMAN, J.M.; DAVIS, M.B.; HOMMER DW. Greater Activation in Left Hemisphere Language-Related Regions During Simple Judgment Tasks Among Substance-Dependent Patients in Treatment for Alcoholism. Alcohol: Clin Exp Res, v. 34, n. 2, 331-41, 2010.

GODOY, S. et al. Concepções teóricas acerca das funções executivas e das altas habilidades. Cadernos de Pós-Graduação em Distúrbios do Desenvolvimento, São Paulo, v.10, n.1, 2010.

GOLD, M. S.; ADAMEC, C. The encyclopedia of alcoholism and alcohol abuse. New York: Infobase, 2010. 
GOLDSTEIN, R.Z.; VOLKOW, N.D. Drug Addiction and its Underlying Neurobiological Basis: Neuroimaging Evidence for the Involvement of the Frontal Cortex. Am JPsychiatry, 159, 1642-52, 2002.

GOUDRIAAN, A.E.; OOSTERLAAN, J.; BEURS, E.D.; BRINK, W.V. Neurocognitive functions in pathological gambling: a comparison with alcohol dependence, Tourette syndrome and normal controls. Addiction, v.101, n.4, p. 534-47, 2006.

GRUBER, S.A.; YURGELUN-TODD, D.A. Neuropsychological Correlates of Drug Abuse. In Kaufman MJ (ed.) Brain Imaging in Substance Abuse: Research, Clinical and Forensic Applications, New Jersey, Humana Press, p. 199-229, 2001.

HILDEBRANDT, H.; BROKATE, B.; ELING, P.; LANZ, M. Response shifting and inhibition, but not working memory, are impaired after long-term heavy alcohol consumption. Neuropsychology, 18, 203-211, 2004.

HOUSTON, R. J.; DERRICK, J. L.; LEONARD, K. E.; TESTA, M.; QUIGLEY, B. M. \& KUBIAK, A. Effects of heavy drinking on executive cognitive functioning in a community sample. Addictive Behaviours, 39, p. 345-349, 2014.

JOHNSON CA, XIAO L, PALMER P, SUN P, WANG Q, WEI Y, ET AL. Affective decision-making deficits, linked to a dysfunctional ventromedial prefrontal cortex, revealed in 10th grade Chinese adolescent binge drinkers. Neuropsychology, 46, 714-26, 2008.

KAPLAN, H. I.; SADOCK, B. J.; GREBB, J. A. Compêndio de psiquiatria: ciências do comportamento e psiquiatria clínica. 7. ed. Porto Alegre: Artmed. 1997.

KAREKEN, D. A. ET AL. Family history of alcoholism interacts with alcohol to affect brain regions involved in behavioral inhibition. Psychopharmacology, p.1-11, 2013.

KIM, Y. T.; SOHN, H. \& JEONG, J. Delayed transition from ambiguous to risky decision making in alcohol dependence during lowa Gambling Task. Psychiatry Research, 190(2-3), 297-303, 2011.

KOPERA, M., WOJNAR, M., BROWER, K., GLASS, J., NOWOSAD, I., GMAJ, B. \& SZELENBERGER, W. (2012). Cognitive functions in abstinent alcohol-dependent patients. Alcohol, v. 46, n. 7, p. 665-671, 2012.

KRISTENSEN, C. H. Funções executivas e envelhecimento. In M. A. M. P. PARENTE (Ed.), Cognição e envelhecimento (pp. 97-111). Porto Alegre: Artmed, 2006. 
LANGLAIS, P.J. \& CICCIA, R.M. An examination of the synergistic interaction of ethanol and thiamine deficiency in the development of neurological signs and long-term cognitive and memory impairments. Alcohol Clinical Experimental Research, 25, 622-634, 2000.

LARANJEIRA R, NICASTRI S. Abuso e dependência de álcool e drogas. In: ALMEIDA O, DRACTU L, LARANJEIRA R, Manual de psiquiatria. Rio de Janeiro: Guanabara Koogan, 1996. p. 84-9.

LARANJEIRA R, PINSKY I, ZALESKI M, CAETANO R, DUARTE PCAV, SANCHES M, et al. I Levantamento nacional sobre os padrões de consumo de álcool na população brasileira [online]. Brasília (DF): Secretaria Nacional Antidrogas; 2007 [acesso 2018 Ago 12]. Disponível em:

http://bvsms.saude.gov.br/bvs/publicacoes/relatorio padroes consumo al cool.pdf.

LONGENECKER, G. Como agem as drogas: abuso de drogas e o corpo humano. São Paulo, Quark, 1998.

LORING, D. (Ed.). INS Dictionary of neuropsychology. New York: Oxford University Press, 1999.

LYVERS, M.; HASKING, P.; HANI, R.; RHODES, M. \& TREW, E. (2010). Drinking motives and drinking behavior among young adults. Addictive Behaviors, v.35, n. 2, p. 116- 122, 2010.

MALLOY-DINIZ, L. F.; FUENTES, D.; MATTOS, P. \& ABREU, N. Avaliação Neuropsicológica. Porto Alegre, Artmed, 2010.

MALLOY-DINIZ, L. F.; SEDO, M.; FUENTES, D. \& LEITE, W. B. Neuropsicologia das funções executivas. In D. FUENTES, L. F. MALLOYDINIZ, C. H. P. CAMARGO \& R. M. COSENZA (Eds.), Neuropsicologia: teoria e prática. Porto Alegre: Artmed, 2008.

MITCHELL, R.L.C. \& PHILLIPS, L.H. The psychological, neurochemical, and functional neuroanatomical mediators of the effects of positive and negative mood on executive functions. Neuropsychologia. 45, 617-629, 2007.

MLLER, EARL \& D. WALLIS, JONATHAN. Executive function and higherorder cognition: definition and neural substrates. encyc. neurosci. 4, 99104, 2009.

NASSIF, S. L. S.; ROSA, J. T. Cérebro, inteligência e vínculo emocional na dependência de drogas. São Paulo: Vetor. 2003.

NATIONAL INSTITUTE OF HEALTH. Alcohol alert (NIAA Publication No 63). U.S Department of Health \& Human Services, 2004. 
Noel, X. \& Bechara, A. Response Inhibition Deficit Is Involved in Poor Decision Making Under Risk in Nonamnesic Individuals With Alcoholism. Neuropsychology, 21 (6), 778-786, 2007.

NOËL, X.; BECHARA, A.; D'ACREMONT, M.; VAN DER LINDEN, M.; HANAK, C.;VERBANCK, P. Response inhibition deficit is involved in poor decision making under risk in nonamnesic individuals with alcoholism. Neuropsychology, v.21, n.6, p.778, 2007.

OLIVEIRA, M.S.; LARANJEIRA, R.; JAEGER, A. Estudo dos prejuízos cognitivos na dependência do álcool. Psicol Saúde Doenças, v.3, n. 2, p. 205-12, 2002.

ORGANIZACAO MUNDIAL DE SAUDE, Classificacao de transtornos mentais e de comportamento da CID-10, Porto Alegre: Artmed, 1992.

PAPAZIAN, O.; ALFONSO, I. \& LUZONDO, R. J. Trastornos de las funciones ejecutivas. Revista de Neurologia, v. 42, n. 3, p. 45-50, 2006.

PARSONS, O. A. Neurocognitive deficits in alcoholics and social drinkers: a continuum? Alcoholism, Clinical and Experimental Research, v. 22, n. 4, 954-61, 1998.

PFEFFERBAUM, A.; SULLIVAN, E.V. \& ROSENBLOON, M.J. Pattern of motor and cognitive deficits in detoxified alcoholic men. Alcohol Clinical Experimental Research. 25, 611-621, 2000.

PIEK, J.P. et al. The relationship between motor coordination, executive functioning and attention in school aged children. Archives of Clinical Neuropsychology, 19, 1063-1076, 2004.

PINEL, J.P. Biopsicologia. Porto Alegre: Artmed, 2005.

PLISZKA, S.R. Neurociência para o clínico de saúde mental.Porto Alegre: Artmed, 2004.

POWELL, K. B. \& VOELLER, K. K. S. Prefrontal executive function syndromes in children. Journal of Child Neurology, 19, 785-797, 2004.

RAMAEKERS, JOHANNES G. et al. Tolerance and cross-tolerance to neurocognitive effects of THC and alcohol in heavy cannabis users. Psychopharmacology, v.214, n.2, p. 91-401, 2011.

RAMOS, S.; BERTOLOTE, J. 0 alcoolismo hoje. $3^{\text {a }}$ ed. Porto Alegre, Artes Médicas, 1997.

RAPPAPORT C. Encarando a adolescência. São Paulo (SP): Ática; 1995. 
RIGONI, M. S. et al. Alcoolismo e Avaliação de Funções Executivas: Uma Revisão Sistemática. Porto Alegre, Psico-PUCRS, v. 44, n. 1, jan./mar., p. 122-129. 2013.

ROCA, M.; PARR, A.; THOMPSON, R.; WOOLGAR, A.; TORRALVA, T.; ANTOUN, N.M.; MANES, F. \& DUNCAN, J. Executive function and fluid intelligence after frontal lobe lesions. Brain : a journal of neurology, 133, p.234-47, 2010.

ROCHA, L.A. et al. Consumo de álcool entre estudantes de faculdades de Medicina de Minas Gerais, Brasil. Rev bras educ med.; 35(3): 369-375. 2011.

ROTTA, N. (2006). Dispraxias. In N. ROTTA; L. OHLWEILER \& R. RIESGO (Eds.) Transtornos da aprendizagem: Abordagem neurobiológica e multidisciplinar. Porto Alegre, Artmed, p. 207-220, 2006.

SANTOS, F. H. Funções executivas. In V. M. ANDRADE \& F. H. SANTOS \& O. F. A. BUENO (Eds.), Neuropsicologia hoje. São Paulo, Artes Médicas, 2004.

SCAIFE, J. C., \& DUKA, T. (2009). Behavioural measures of frontal lobre function in a population of young social drinkers with binge drinking pattern. Pharmacology, Bio-chemistry, and Behavior, 93, p. 354-362, 2009.

SÉGUIN, J. R.; ARSENEAULT, L. \& TREMBLAY, R. E. The contribution of "cool" and "hot" components of decision-making in adolescence: Implications for developmental psychopathology. Cognitive Development. 22 (4), 530-543, 2007.

SOURNIA, J. C. Histoire de l'alcoolisme. Paris: Flammarion, 1986.

THOMA, PATRIZIA et al. Mental state decoding and mental state reasoning in recently detoxified alcohol-dependent individuals. Psychiatry research, 2012.

THUSH, C.; WIERS, R.W.; AMES, S.L., GRENARD, J.L.; SUSSMAN, S. \& STACY, A.W. Interactions between implicit and explicit cognition and working memory capacity in the prediction of alcohol use in at-risk adolescents. Drug and Alcohol Dependence, 94, p.116-124, 2008.

TOMASSINI, A.; STRUGLIA, F.; SPAZIANI, D.; PACIFICO, R.; STRATTA, P. \& ROSSI, A. Decision Making, Impulsivity, and Personality Traits in Alcohol-Dependent Subjects. The American Journal on Addictions, 21, n.3, p. 263-267, 2012. 
TONIETTO, L. et al. Interfaces entre funções executivas, linguagem e intencionalidade. Paidéia (Ribeirão Preto), Ribeirão Preto, v. 21, n. 49, p. 247-255, Aug. 2011. Available from <http://www.scielo.br/scielo.php?script=sci_arttext\&pid=S0103863X2011000200012\&Ing=en\&nrm=iso>. access on 02 Oct. 2018. http://dx.doi.org/10.1590/S0103-863X2011000200012.

UEKERMANN, J; DAUM, I.; SCHLEBUSCH, P.; WIEBEL, B., \& TRENCKMANN, U. Depression and cognitive functioning in alcoholism. Addiction, 98 (11), 1521-9, 2003.

; CHANNON, S.; WINKEL, K.; SCHLEBUSCH, P. \& DAUM, I. Theory of mind, humour processing and executive functioning in alcoholism. Addiction, 102 (2), 232-40, 2007.

VERDEJO-GARCIA, A., LOPEZ-TORRECILLAS, F., ARCOS, F. A., \& PEREZ-GARCIA, M. Differential effects of MDMA, cocaine, and cannabis use severity on distinctive components of the executive functions in polysubstance users: A multiple regression analysis. Addictive Behaviors, 30, 89-101, 2005.

VIALA-ARTIGUES, J. \& MECHETTI, C. Histoire de I'alcool archéologie partie 1, 2003.

WASHTON, A. M., \& ZWEBEN, J. E. Prática psicoterápica eficaz dos problemas com álcool e drogas. (M. Armando, Trad.). Porto Alegre: Artmed, 2009 (Original publicado em 2006).

WOICIK, P.A.; STEWART, S.H.; PIHL, R.O.; CONROD, P.J. The substance use risk profile scale: A scale measuring traits linked to reinforcement-specific substance use profiles. Addictive Behaviors, 34, p.10421055, 2009.

WORLD HEALTH ORGANIZATION - WHO. Global status report on alcohol. Genebra:WHO, 2017.

ZHANG, L., WELTE, J. \& WIECZOREK, W. The role of aggression related alcohol expectancies in explaining the link between alcohol and violent behavior. Substance Use and Misuse, 37(4),457-471, 2002.

ZIN, S.; STEIN, R.; SWARTZWELDER, H.S. Executive Functioning Early in Abstinence From Alcohol. Alcohol: Clin Exp Res, v. 28, n. 9, p. 133846, 2004. 